\title{
Does methylene blue help in the early identification of the recurrent laryngeal nerve?
}

\section{Chhetri SS ${ }^{1}, \mathrm{KC} \mathrm{T}^{2}$, Bista $\mathrm{M}^{3}$, Mahato $\mathrm{NB}^{4}$}

${ }^{1}$ Sujan Singh Chhetri, Assistant Professor; ${ }^{2}$ Toran KC, Professor; ${ }^{3}$ Meera Bista, Associate Professor; ${ }^{4}$ Nayan Bahadur Mahato, Lecturer; Department of ENT-HNS, Kathmandu Medical College Teaching Hospital, Sinamangal, Kathmandu, Nepal

\begin{abstract}
Background: Identification of recurrent laryngeal nerve is of utmost importance during thyroid surgery. Different anatomical landmarks have been used to recognize and preserve the nerve. Injury may lead to vocal cord paralysis. Different adjuvant methods have been used to aid in the identification of the nerve.

Objective: To determine whether methylene blue smear helps to identify the recurrent laryngeal nerve safely and efficiently.

Methodology: Observational cross sectional study done in 30 patients who underwent different thyroidectomies within a duration of one year. Recurrent laryngeal nerve identified using methylene blue smear and compared with the conventional visualization techniques exercising different known anatomical landmarks. The duration and ease of identification of the nerve was noted and graded.

Results: Total of 39 recurrent laryngeal nerves were identified from 28 females and two males. The duration and the ease of dissection of the nerve was inconstant. Earliest time for recognition of the nerve was one and half minutes while the slowest time was 12 minutes.

Conclusion: No matter what techniques are used, visual identification and verification of the recurrent laryngeal nerve is of paramount importance. No techniques demonstrated added advantage to one another.
\end{abstract}

Key words: Ancillary technique; Methylene blue smear; Recurrent laryngeal nerve.

DOI: http://dx.doi.org/10.3126/jkmc.v7i1.20622

\section{INTRODUCTION}

T hyroid Surgery is one of the most frequently done Head and Neck surgeries'. Complications after thyroid surgery are not uncommon. Injury to the recurrent laryngeal nerve (RLN) is a significant risk associated with thyroid surgery. Though such incidence is low, when it does occur, it is not without morbidity ${ }^{2}$. It has a very disastrous effect especially in cases of bilateral thyroid surgery.

As the surgeons are aware of this, they have identified various important anatomical structures and come up with different dissection techniques to preserve the nerve intact.

\section{Address for correspondence}

Dr. Sujan Singh Chhetri,

Assistant Professor, Department of ENT-HNS

Kathmandu Medical College Teaching Hospital, Sinamangal, Kathmandu

Email: sujansu_chhetri@hotmail.com
Anatomical identification has always been endorsed as the safest method of identifying the nerve during the surgery. Still at times, they find it very frustrating to identify it. The use of methylene blue smear yields to distinguish the nerve from the surrounding important structures and may prove to be noteworthy choice for the ease of identification of the nerve. It is based upon the fact that neuron oxidized reduce methylene blue more rapidly than other cell types. Reduced solutions are more transparent or yellowish ${ }^{3}$.

Methylene blue is factory sterilized and is bactericidal ${ }^{4}$. It has also been used previously in abdominal cavities by various surgeons and has also been used intravenously for different medical conditions. Methylene Blue has been used previously in several studies for identification of parathyroid glands ${ }^{5}$.

Infusion of methylene blue has rendered identification of the gland stress-free. Similar studies are sparse in cases of identification of the recurrent laryngeal nerve. Infusion is not without post-operative discomforts. This 
study focuses on whether the use of Methylene Blue as a smear helps to identify the recurrent laryngeal nerve efficiently and safely.

\section{METHODOLOGY}

This is an observational cross-sectional study done at the Department of ENT-HNS, Kathmandu Medical College, Sinamangal, Kathmandu, Nepal. The duration of study was twelve months (January 2016 - December 2017). Ethical clearance was obtained from the Institutional Review Committee of Kathmandu Medical College. Informed consent was taken from each patient.

Patients who were to undergo different thyroid surgeries for different pathological conditions were included. But patients with history of previous thyroid surgeries, vocal cord paralysis were excluded. All patients posted for surgery underwent fibreoptic nasopharyngolarygoscopic examination to assess vocal cord mobility before and after the surgery.

Altogether, 30 patients were subjected to different thyroidectomy procedures according to the need of the pathology and involvement of the thyroid gland.Total of 39 recurrent laryngeal nerves were identified and compared. After identification and ligation of middle thyroid vein and before the ligation of the inferior pole of thyroid, $0.5 \mathrm{ml}$ of methylene blue was taken in a syringe and smeared along the perilobar area, kept for 15 seconds and swabbed. Dissection was done to identify the recurrent laryngeal nerve and its relation with the inferior thyroid artery.The ease of identification of the nerve effectively and reliably was based on the timing required to identify the nerve and also on the effort and difficulties encountered during the procedure by different surgeons.The level of difficulty was graded as easy if the nerve was identified within five minutes of smearing otherwise difficult if it took more than five minutes. With the exception of the hemithyroidectomy, the simplicity and timing required to identify the nerve on the both sides were compared.

The data collected were analyzed with Statistical Package for Social Sciences (SPSS) version 20, MS Excel. Paired T test was applied and $p<0.05$ considered significant.

\section{RESULTS}

Of the total 30 patients, 28 (93\%) were female and only two(7\%) were male. 21 Hemithyroidectomy, three Subtotal Thyroidectomy, two Near Total Thyroidectomy and four Total Thyroidectomy were done. Total of 39 recurrent nerves were identified altogether.

As a whole, among the methylene blue smeared group, the shortest duration to identify the recurrent laryngeal nerve was two minutes and the longest duration was 12 minutes. Whereas in the conventional technique, where it was identified using various anatomical landmarks, the quickest time was one and a half minute and lengthiest was ten minutes.

Among the Hemithyroidectomy group, 11 recurrent laryngeal nerves were smeared with methylene blue before dissection whereas, 10 were identified by conventional dissection techniques (Table 1). The difference is considered to be not statistically significant $(p=0.3290)$.

Table 1: Time taken to identify RLN in Hemithyroidectomy group

\begin{tabular}{lcc} 
& \multicolumn{2}{c}{ Hemithyroidectomy } \\
& Smeared & Not Smeared \\
$\begin{array}{l}\text { Mean duration } \\
\text { (minutes) }\end{array}$ & 4.591 & 3.75 \\
SD & 2.107 & 1.814 \\
SEM & 0.635 & 0.574 \\
\hline
\end{tabular}

$\mathrm{SD}=$ Standard Deviation; $\mathrm{SEM}=$ Standard error of mean

Table 2: Time taken to identify RLN in different thyroidectomy groups

\begin{tabular}{|c|c|c|c|c|c|c|}
\hline & \multicolumn{2}{|c|}{ Subtotal Thyroidectomy } & \multicolumn{2}{|c|}{ Near Total Thyroidectomy } & \multicolumn{2}{|c|}{ Total Thyroidectomy } \\
\hline & Smeared & Not Smeared & Smeared & Not Smeared & Smeared & Not Smeared \\
\hline Mean duration (minutes) & 6.67 & 6.33 & 5.5 & 5.5 & 3.5 & 5 \\
\hline SD & 5.03 & 3.21 & 3.54 & 2.12 & 1 & 1.41 \\
\hline \multirow[t]{2}{*}{ SEM } & 2.91 & 1.86 & 2.5 & 1.5 & 0.5 & 0.71 \\
\hline & \multicolumn{2}{|c|}{$p=0.8075$} & \multicolumn{2}{|c|}{$\mathrm{p}=1$} & \multicolumn{2}{|c|}{$p=0.2967$} \\
\hline
\end{tabular}

$\mathrm{SD}=$ Standard Deviation; $\mathrm{SEM}=\mathrm{Standard}$ error of mean 
With other types of thyroidectomy, the mean duration for the identification of the recurrent laryngeal nerve in both the groups are shown in table 2. None of the difference were statistically significant.

Figure 1 shows the obvious left recurrent laryngeal nerve from the surrounding structures after gentle dissection in the tracheoesophageal groove subsequently following $0.5 \mathrm{ml}$ of methylene blue smear. The nerve was identified along its course up to Berry's ligament with preservation of inferior thyroid artery. None of the patient had vocal cord palsy post-operatively.

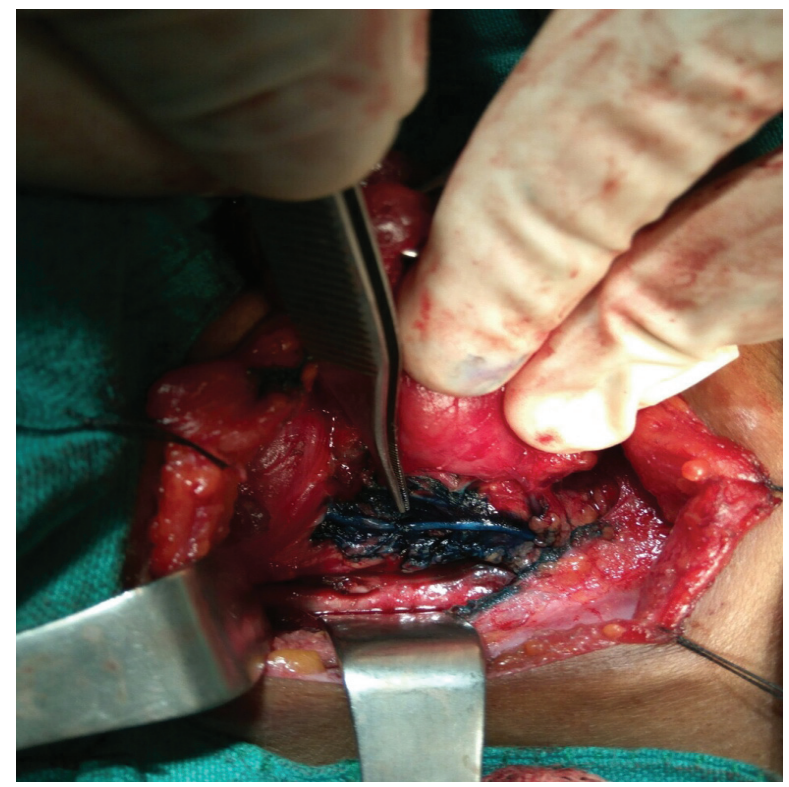

Figure 1: Identification of Recurrent Laryngeal Nerve after Methylene Blue smear.

\section{DISCUSSION}

Different anatomical landmarks, boundaries have been devised to ensure the safety of the recurrent laryngeal nerve during thyroid surgery. Every so often surgeons face difficulties in identifying this framework leading to risk of injury to the nerve and devastating consequences thereafter. The simple technique of methylene blue smear may avoid all such complications and reduce morbidity.

Since the discovery of the recurrent nerves by Galen during the second century, the later anatomists and surgeons had great respect for the nerve. It's anatomical location and importance were highlighted. In 1938, Dr. Frank Lahey explained the indulgent and careful, delicate dissection of recurrent laryngeal nerve during thyroidectomy ${ }^{6}$.
Anatomy of RLN appears more constant along its distal segment near the cricothyroid joint. Shindo ML et $\mathrm{al}^{7}$ demonstrated that approaching it along its distal portion was safe and effective. Potential advantages included less chance of disrupting blood supply to inferior parathyroids and lesser variability of the nerve. The application of the stain helped to hasten the identification of the nerve from the surrounding structures as compared to the anatomical exploration.

Injection of a blue dye in the inferior thyroid artery when the identification of the recurrent laryngeal nerve was elusive proved to be a useful method in a study done by Gulcin Hepgulet $\mathrm{al}^{8}$. However, it was emphasized that it was not a substitution for a conventional visual identification of the nerve. Comparably, when the methylene blue was smeared along the perilobar area before the beginning of the dissection, at times it was stress-free to identify the nerve but was not superior to the conventional technique.

Occasional difficulties encountered during dissection even after smearing by the stain and relatively easy identification of the nerve equally after conventional technique proves to show no definite benefits in regards to morbidity.In a center with high surgical volume, cautious identification of recurrent laryngeal nerve regardless of the procedure done, failed to reduce the morbidity. The incidence of temporary and permanent recurrent laryngeal nerve palsy rates was $2.9 \%$ and $0.5 \%$ respectively 9 .

Chiang FY et al described a more reliable and safer way to preserve all branches of the RLN. Identification, verification and documentation of the integrity of the nerve intra-operatively with intra-operative neuromonitoring was recommended ${ }^{10}$. Since the cost of the device remains very high, every centers that perform thyroidectomy cannot afford it and relies on other techniques of identification.

Smith Jet al observed temporary and permanent RLN palsy rate of $6.1 \%$ and $1.7 \%$ respectively. Even with intraoperative neural monitoring and stimulation following dissection did not help in determining the fate of RLN injuries ${ }^{11}$. There is a lack of clear evidence that the increased use of neural monitoring system has led to minimizing the RLN injuries ${ }^{12}$.

Nyeki ARN et $\mathrm{al}^{13}$ considered various anatomical landmarks for the identification of the recurrent laryngeal nerve in relation to different important surrounding structures. He also highlighted the variability of the 
course of the nerve which made it even more problematic. He suggested that no structures could solely be regarded and visualization was extremely important. Likewise, considering the course of the nerve, smearing was no superior to the conventional techniques applied. It all depended on the careful and meticulous dissection to appreciate the nerve itself.

\section{REFERENCES}

1. Fortuny VJ, Guigard S, Karenovics W, Triponez F. Surgery of the thyroid: recent developments and perspective. Swiss Med Wkly. 2015;28: 145. w14144. doi: 10.4414/smw.2015.14144. eCollection 2015

2. Thomusch O, Machens A, Sekulla C,Ukkat J, Lippert $\mathrm{H}$, Gastinger I, et al.Multivariate analysis of risk factors for postoperative complications in benign goiter surgery: prospective multicenter study in Germany. World J Surg. 2000;24(11):1335-41.

3. Murat O, Lorke DE, Hasan Md, Petroianu GA. Cellular and Molecular Actions of Methylene Blue in the Nervous System. Med Res Rev. 2011; 31(1): 93-117. doi:10.1002/med.20177.

4. Methylene Blue Anhydrous. National Center for Biotechnology Information. [Internet] PubChem Compound Databse; CID=6099, [cited 2017 Oct 4] http://pubchem.ncbi.nlm.nih.gov/ compound/6099.

5. Derom AF, Wallaert PC, Janzing HM, Derom FE. Intraoperative identification of parathyroid glands with methylene blue infusion. Am J Surg. 1993; 165(3): 380-2.

6. Kaplan EL, Salti GI, Roncella M, Fulton N, Kadowaki $M$. History of the recurrent laryngeal nerve: from Galen to Lahey. World J Surg. 2009; 33(3): 386-93. doi: 10.1007/s00268-008-9798-z.

7. Shindo ML, Wu JC, Park EE. Surgical anatomy of the recurrent laryngeal nerve revisited. Otolaryngol Head Neck Surg. 2005;133(4):514-9.

\section{CONCLUSION}

Regardless of the methods used, visual identification of the recurrent laryngeal nerve remains the main factor determining the preservation of the nerve and to decrease the incidence of post-operative recurrent laryngeal nerve palsy. No such techniques are grander. Methylene Blue smear can be a mode of an ancillary tool but cannot be always reliant on.
8. Hepgul G, Kucukyilmaz M, Koc O, Duzkoylu Y,Sari YS, Erbila Y. The identification of recurrent Laryneal Nerve by injection of blue Dye into the Inferior Thyroid Artery in Elusive Locations. J Thyroid Res. 2013;2013: Article ID 539274, 3 pages. doi:10.1155/2013/539274.

9. Bergamaschi R, Becouarn G, Ronceray J, Arnaud JP. Morbidity of thyroid surgery. Am J Surg. 1998;176(1): 71-5.

10. Chiang FY, Lu IC, Chen HC, Chen HY, Tsai CJ, Hsiaso PJ, et al. Anatomical Variation of Recurrent Laryngeal Nerve During Thyroid Surgery: How to identify and handle the variations with intraoperative neuromonitoring. Kaohsiung J Med Sci. 2010; 26:575-83.

11. Smith J, Douglas J, Smith B, Dougherty T, Ayshford C. Assessment of recurrent laryngeal nerve function during thyroid surgery. Ann R Coll Surg Engl. 2014; 96:130-5. doi: 10.1308/00358841X13814021676594

12. Goretzki PE, Schwarz K, Brinkman J, Wirowski D, Lammers BJ. The impact of intraoperative neuromonitoring (IONM) on surgical strategy in bilateral thyroid diseases: is it worth the effort? World J Surg. 2010; 34: 1274-84.

13. Ngo Nyeki AR, Njock LR, Miloundja J, EveheVokwely $J E$, Bengono G. Recurrent laryngeal nerve landmarks during thyroidectomy. Eur Ann Otorhinolaryngol Head Neck Dis. 2015; 132(5): 265-9.doi: 10.1016/j. anori.2015.08.002. 